\title{
Étude comparée par la méthode des sachets de nylon de la dégradation de la paille de blé dans le rumen et le cæcum des ovins et des caprins
}

\author{
ZM Bicaba, PE Arista, F Faurie, C Masson, JL Tisserand \\ ENSSAA-INRA, laboratoire de recherches de la chaire de zootechnie, \\ 26, bd Dr-Petitjean, 21000 Dijon, France
}

\begin{abstract}
Summary - Compared degradation of wheat straw in the rumen and in the caecum of sheep and goats by the in sacco method. A study of in sacco $(24 \mathrm{~h})$ cellulolytic activity in the rumen and caeca of 3 rams and 3 goats showed that there was no real difference between the 2 digestive behaviours. Cellulolytic activity turned out to be higher in the goats' rumens as compared to the sheep and more particularly with nitrogen deficient diets.
\end{abstract}

Dans le but d'évaluer la part de l'activité microbienne du gros intestin dans la digestion des fourrages à teneur élevée en parois chez les ovins et les caprins, nous avons comparé la dégradation de la paille de blé dans le rumen et dans le cæcum des 2 espèces.

Matériel et méthodes - Trois boucs et 3 béliers adultes castrés, pesant en moyenne respectivement 50 et $60 \mathrm{~kg}$, porteurs de canules permanentes du rumen et du cæcum, sont maintenus en cages individuelles. Ils reçoivent successivement 3 rations composées chacune de $800 \mathrm{~g}$ de paille, et, soit de $300 \mathrm{~g}$ de maïs (grains) $+104 \mathrm{~g}$ de tourteau de soja (P1), soit de $350 \mathrm{~g}$ de maïs $+22 \mathrm{~g}$ d'ure $(\mathrm{r} 2)$, soit de $350 \mathrm{~g}$ de maïs (P3). Ces rations sont distribuées au niveau de l'entretien moyen des ovins et des caprins, 2 fois par jour, à $8 \mathrm{~h}$ et $16 \mathrm{~h}$. Les animaux disposent à volonté d'eau et de blocs riches en minéraux. La dégradation de la matière sèche (MS) est mesurée dans le rumen et le cæcum par une méthode des sachets de nylon dérivée de celle décrite par Demarquilly et Chenost (1969) et adaptée par Alrahmoun (1985). Les sachets contiennent de la paille de blé broyée (broyeur à marteaux; tamis de $0,8 \mathrm{~mm}$ ) à raison de $3 \mathrm{~g}$ par sachet placé dans le rumen des moutons et de $0,8 \mathrm{~g}$ par sachet placé dans le rumen des caprins, et dans les cæcum. Le rapport: poids de paille/surface du sachet, est le même : $0,06 \mathrm{~g} / \mathrm{cm}^{2}$. La composition chimique de la paille est (\% de MS) : $M M=8,6 ;$ MAT $=3,8$; $\mathrm{NDF}=79,7 ;$ ADF $=50,3 ;$ lignine $=7,8$.

Au cours de chaque période, 3 sachets sont introduits dans le rumen $(R), 2$ sachets dans le rumen puis le cæcum du même animal $(R+C e)$, et 2 sachets dans le cæcum (Ce). La mesure est répétée. La durée du séjour des sachets dans chaque site est de $24 \mathrm{~h}$. Les résultats moyens par espèce, par régime et par site sont comparés par le test $t$ de Student.

Résultats et discussion - La dégradation de la MS dans le rumen est plus élevée chez les caprins que chez les ovins, quel que soit le régime 
(tableau I). Les valeurs de la P3 confirment les résultats obtenus précédemment dans notre Laboratoire (Tisserand et al, 1986) et par d'autres chercheurs (Obara et Shimbayashi, 1987; Djajanegara et Doyle, 1989), qui concluent que les ovins sont plus sensibles à la carence azotée que les caprins. Les caprins, grâce sans doute à une meilleure utilisation de l'azote (recyclage de l'urée surtout), réduisent le déficit azoté au niveau du rumen, principalement, et du cæcum dans une moindre mesure, ce qui permet une activité microbienne plus efficace.

Nos résultats mettent aussi en évidence un effet de la nature du complément azoté : l'activité microbienne dans le cæcum semble indépendante de la nature et de la quantité du complément azoté chez les caprins, alors qu'elle est diminuée chez les ovins avec le régime P3 sans complément azoté. Cela confirme les travaux antérieurs qui mettent en évidence un meilleur recyclage de l'N chez les caprins. Toutefois, nos résultats sont à considérer avec prudence car le cæcum ne constitue qu'une partie du gros intestin et le temps de séjour des aliments dans ce compartiment, arbitrairement fixé à $24 \mathrm{~h}$, ne correspond vraisemblablement pas à la durée réelle. En effet, pour ce qui concerne le gros intestin, le temps de séjour apparaît plus élevé chez les caprins que chez les ovins (Alrahmoun, 1985).

En conclusion, et malgré les réserves précédentes, cet essai met en évidence que l'activité cellulolytique dans le cæcum est presque équivalente à celle du rumen et que l'activité cellulolytique du rumen est plus élevée chez les caprins que chez les ovins, surtout avec les régimes pauvres en azote.

Alrahmoun W (1985) Thèse d'état, université de Dijon, $213 p$

Demarquilly C, Chenost M (1969) Ann Zootech 18, 419-435

Djajanegara A, Doyle PT (1989) Anim Feed Sci Technol 27, 31-47

Obara Y, Shimbayashi K (1987) Jpn $J$ Zootech Sci 58, 611-617

Tisserand JL, Bellet B, Masson C (1986) Reprod Nutr Dév 26, 313-314

Tableau I. Disparition de la MS de la paille après $24 \mathrm{~h}$ de séjour dans le rumen puis/ou dans le cæcum (moyenne \pm écart type).

\begin{tabular}{lllcl}
\hline Période & Espèce & Rumen $(R)$ & $\begin{array}{c}\text { Rumen puis cæcum } \\
(R+C e)\end{array}$ & Cæcum (Ce) \\
\hline P1 & ovine & $25,3 \pm 2,1^{\mathrm{a}}$ & $42,0 \pm 2,5$ & $25,6 \pm 3,0^{\mathrm{c}}$ \\
& caprine & $29,8 \pm 2,1^{\mathrm{b}}$ & $40,8 \pm 3,5^{\mathrm{d}}$ & $22,7 \pm 2,6^{\mathrm{d}}$ \\
P2 & ovine & $26,8 \pm 3,2^{\mathrm{c}}$ & $37,6 \pm 4,2^{\mathrm{c}}$ & $24,3 \pm 2,5$ \\
& caprine & $29,5 \pm 4,4^{\mathrm{d}}$ & $40,8 \pm 2,4^{\mathrm{d}}$ & $25,1 \pm 1,8$ \\
P3 & ovine & $20,9 \pm 1,5^{\mathrm{a}}$ & $33,9 \pm 1,1^{\mathrm{a}}$ & $22,7 \pm 2,6$ \\
& caprine & $26,1 \pm 4,0^{\mathrm{b}}$ & $37,5 \pm 2,7^{\mathrm{b}}$ & $22,4 \pm 1,7$ \\
\hline
\end{tabular}

Pour une même période et un mème site, les moyennes indicées diffèrent significativement; ${ }^{a}{ }^{b}: P<0,01 ;{ }^{c}{ }^{d}: P<0,05$. 